\title{
Intorno alla risoluzione razionale di una classe di equazioni algebriche fra quattro variabili.
}

\author{
(Di Federigo Exriques, a Bologna.)
}

1. Scopo di questa Nota è d'indicare un criterio generale per la possibilità di risolvere razionalmente una classe di equazioni algebriche fra quattro variabili.

I. Sia $f\left(x_{1} x_{2} x_{3} x_{4}\right)=0$ un'equazione algebrica, irriducibile, di grado 2 complessivamente rispetto alle variabili $x_{1} x_{2}$, e di grado qualsiasi $n$ rispetto ad $x_{3} x_{4}$, affinchè l'equazione $f=0$ possa esser risoluta razionalmente, cioè 1onendo $x_{1} x_{2} x_{z_{1}} x_{4}$ funzioni razionali (in generale non razionalmente invertibili) di tre parametri:

$$
\begin{aligned}
& x_{1}=\varphi_{1}\left(u_{1} u_{2} u_{3}\right) \\
& x_{2}=\varphi_{2}\left(u_{1} u_{3} u_{3}\right) \\
& x_{3}=\varphi_{3}\left(u_{1} u_{2} u_{3}\right) \\
& x_{1}=\varphi_{1}\left(u_{1} u_{2} u_{3}\right)
\end{aligned}
$$

basta che si possano determinare quattro funzioni razionali di due variabili

$$
\begin{aligned}
& x_{1}=x_{1}\left(v_{1} v_{1}\right) \quad x_{2}=x_{2}\left(v_{1} v_{2}\right) \\
& x_{3}=x_{3}\left(v_{1} v_{2}\right) \quad x_{4}=x_{4}\left(v_{1} v_{2}\right),
\end{aligned}
$$

per modo che $x_{3} x_{4}$ non siano legate fra loro da una relazione indipendente $d a v_{1} v_{2}$ e che soddisfino all'equazione $f\left(x_{1} x_{2} x_{3} x_{4}\right)=0$.

In linguaggio geometrico:

l'. Una varietà di 3 dimensioni d'ordine $n>2$, in $S_{4}$, dotata d'una retta $(n-2)$-pla, a, si può rappresentare sopra un'involuzione di $S_{3}$, se contiene una superficie razionale non composta di coniche giacenti in piani per a. 
Più generalmente :

II. Una varietà algebrica di 3 (o di $r>3$ ) dimensioni, possedente una congruenza del 1." ordine di curve razionali, si può rappresentare sopra una involuzione dello spazio $S_{3}$ (o $S_{\text {, }}$ ), se contiene una superficie (o una varietà $V_{r-1}$ ) razionale, non composta di curve della congruenza.

Un'applicazione particolare di questo teorema ci fornisce il seguente corollario:

Ogni congruenza del 1..$^{\circ}$ ordine di curve razionali nello spazio ordinario, $\left(S_{s}\right)$, si può far nascere da una stella di rette per mezzo di una trasformazione razionale (generalmente non invertibile) dello spazio.

Nel caso di curve razionali d'ordine dispari la congruenza può ridursi ad una stella di rette già con una trasformazione birazionale (invertibile). Sappiamo (Montesano) che non è più così quando si tratti di coniche o di curve d'ordine pari. Ora il resultato innanzi enunciato permette di ridurre in generale la costruzione delle congruenze del $1 .^{\circ}$ ordine di curve razionali d'ordine pari, nello spazio, alla classificazione delle involuzioni di JonQuiènes dello spazio e quindi a quella delle involuzioni di gruppi di punti nel piano.

2. Dimostriamo il teorema I'.

Si abbia una varietà $V_{3}^{n}$, d'un certo ordine $n(>2)$, dotata di una retta (n-2)-pla, $a$, nello spazio $S_{4}$; i piani per a segano $V_{3}^{n}$ secondo coniche $C$.

Supponiamo che alla $V_{3}^{n}$ appartenga una superficie razionale $F$, secante le coniche $C$ in $m(\geq 1)$ punti. Riferiamo la $F$ ad una stella di raggi, di centro $O$, in $S_{3}$, per modo che ad ogni punto generico di $F$ corrisponda un raggio per $O$ e viceversa.

Consideriamo una conica $C$ che sega $F$ in $m$ punti: $A_{1}, A_{2}, ., ., A_{m}$. A questi punti rispondono $m$ rette, $a_{1}, a_{2}, \ldots, a_{m}$, per $O$; e così alle coniche $C$ di $V_{3}$ si possono associare gruppi di $m$ raggi, generanti un'involuzione nella stella $O$. Ora si puo porre razionalmente in funzione del punto $A_{i}$, una corrispondenza proiettiva fra la conica $C$ e la retta $a_{i}$. Ne risulta definita razionalmente una corrispondenza (univoca) fra lo spazio $S_{3}$ e la varietà $V_{3}^{n}$, dove ad ogni punto di $S_{3}$ risponde $u n$ punto di $V_{3}^{n}$, e ad ogni punto di $V_{3}^{n}$ risponfono $m$ punti di $S_{3}$; per modo che mentre un punto di $V_{s}^{n}$ si muove descrivendo una conica $C$, gli omologhi $m$ punti in $S_{3}$ descrivono gli $m$ raggi associati a $C$, della stella $O$. Così dunque la $V_{3}^{n}$ viene rappresentata sopra una involuzione di gruppi di $m$ punti in $S_{3}$, c. d. d.

3. Per giungere al teorema piì generale II, basta quindi ricordare che, secondo Noғтнке, ogni curva razionale si può trasformare birazionalmente 
in una conica senza aggiungere irrazionalità numeriche al campo di razionalità definito dai coefficienti della sua equazione, e perciò, come già ebbi luogo di notare (Cfr. Math. Annalen, Bd. 49), ogni varietà $V_{r}$ di dimensione $r$ possedente una congruenza del $1^{\circ}$ ordine di curve razionali, si può trasformare in una varietà $V_{r}^{n}$, d'un certo ordine $n$ in $S_{r+1}$, possedente una retta $(n-2)$-pla.

Il corollario segue immediatamente osservando che se è data nello spazio $S_{3}$ una congruenza di curve razionali, un piano generico di $S_{3}$ porge appunto una superficie razionale non composta colle curve della congruenza.

Bologna, Settembre 1912. 